\section{OPEN ACCESS}

Edited by:

Görsev Yener,

Dokuz Eylul University, Turkey

Reviewed by:

Ayfer Ülgenalp,

Dokuz Eylül University, Turkey

Giovanni Rizzo,

University of Bologna, Italy

${ }^{*}$ Correspondence:

Keith Vosse

kvosse/@mednet.ucla.edu

Specialty section:

This article was submitted to Dementia and Neurodegenerative

Diseases,

a section of the journal

Frontiers in Neurology

Received: 30 August 2020 Accepted: 19 November 2020 Published: 21 December 2020

Citation:

Castro M, Venkateswaran $N$, Peters ST, Deyle DR, Bower M, Koob MD, Boeve BF and Vossel $K$

(2020) Case Report: Early-Onset Behavioral Variant Frontotemporal

Dementia in Patient With

Retrotransposed Full-Length Transcript of Matrin-3 Variant 5

Front. Neurol. 11:600468. doi: 10.3389/fneur.2020.600468

\title{
Case Report: Early-Onset Behavioral Variant Frontotemporal Dementia in Patient With Retrotransposed Full-Length Transcript of Matrin-3 Variant 5
}

\begin{abstract}
Madelyn Castro ${ }^{1}$, Nisha Venkateswaran ${ }^{1,2}$, Samuel T. Peters ${ }^{1}$, David R. Deyle ${ }^{3}$, Matthew Bower ${ }^{4,5}$, Michael D. Koob ${ }^{6}$, Bradley F. Boeve ${ }^{7}$ and Keith Vossel ${ }^{1,2,8 *}$
\end{abstract}

${ }^{1}$ Department of Neurology, N. Bud Grossman Center for Memory Research and Care, University of Minnesota, Minneapolis, MN, United States, '² Department of Neurology, Mary S. Easton Center for Alzheimer's Disease Research at UCLA, David Geffen School of Medicine at UCLA, Los Angeles, CA, United States, ${ }^{3}$ Department of Clinical Genomics, Mayo Clinic Rochester, Rochester, MN, United States, ${ }^{4}$ Division of Genetics and Metabolism, University of Minnesota, Minneapolis, MN, United States, ${ }^{5}$ Molecular Diagnostics Laboratory, M Health-Fairview, University of Minnesota, Minneapolis, MN, United States, ${ }^{6}$ Department of Laboratory Medicine and Pathology, University of Minnesota, Minneapolis, MN, United States, ${ }^{7}$ Department of Neurology, Mayo Clinic Rochester, Rochester, MN, United States, ${ }^{8}$ Institute for Translational Neuroscience, University of Minnesota Medical School, Minneapolis, MN, United States

Frontotemporal dementia (FTD) rarely occurs in individuals under the age of 30 , and genetic causes of early-onset FTD are largely unknown. The current report follows a 27 year-old patient with no significant past medical history presenting with two years of progressive changes in behavior, rushed speech, verbal aggression, and social withdrawal. MRI and FDG-PET imaging of the brain revealed changes maximally in the frontal and temporal lobes, which along with the clinical features, are consistent with behavioral variant FTD. Next generation sequencing of a panel of 28 genes associated with dementia and amyotrophic lateral sclerosis (ALS) initially revealed a duplication of exon 15 in Matrin-3 (MATR3). Whole genome sequencing determined that this genetic anomaly was, in fact, a sequence corresponding with full-length MATR3 variant 5 inserted into chromosome 12, indicating retrotransposition from a messenger RNA intermediate. To our knowledge, this is a novel mutation of MATR3, as the majority of mutations in MATR3 linked to FTD-ALS are point mutations. Genomic DNA analysis revealed that this mutation is also present in one unaffected first-degree relative and one unaffected second-degree relative. This suggests that the mutation is either a disease-causing mutation with incomplete penetrance, which has been observed in heritable FTD, or a benign variant. Retrotransposons are not often implicated in neurodegenerative diseases; thus, it is crucial to clarify the potential role of this MATR3 variant 5 retrotransposition in early-onset FTD.

Keywords: frontotemporal dementia, Matrin 3, case report, retrotransposons, whole genome sequencing 


\section{INTRODUCTION}

Frontotemporal dementia (FTD) refers to a group of dementias characterized by degeneration in the frontal and temporal lobes of the brain (1). Rates of early-onset FTD are high with $13 \%$ of individuals with FTD being under the age of 50 (2). Younger cases of FTD, with onset before the age of 30, tend to exhibit frequent abrupt mood changes, increased aggression, behavioral disinhibition, lack of empathy, and deficits in working memory (3). There are three clinical presentations of FTD: behavioral variant (bvFTD) and two forms of primary progressive aphasia, wherein non-fluent or fluent aphasia are the key neurologic deficits $(4,5)$. In addition, FTD can overlap with other neurodegenerative disease motor deficits including: corticobasal degeneration, progressive supranuclear palsy, and amyotrophic lateral sclerosis (ALS) (6). Approximately $30-50 \%$ of FTD cases have some family history of dementia, parkinsonism or ALS (7), and in $10-20 \%$ a genetic cause is found (8). Positive family history of dementia/parkinsonism/ALS typically shows an autosomal dominant pattern with high penetrance, with at least one firstdegree relative of the proband being affected (7). Mutations in genes encoding microtubule associated protein tau (MAPT), progranulin $(G R N)$, and chromosome 9 open reading frame 72 (C9orf72) account for about half of all familial cases of FTD $(9,10)$. Mutations in MATR 3 , which encodes matrin-3, have been found in some cases of familial FTD as well (11). Matrin-3 is a DNA- and RNA-binding protein that is part of the nuclear matrix and has a wide variety of functions including transcriptional regulation, DNA binding, and RNA splicing and degradation $(11,12)$. Matrin-3 interacts with pathologic markers, such as TAR DNA-binding protein 43 (TDP-43), which aggregates in neuronal cytoplasm in both FTD and ALS (13).

There are several pathological subtypes of FTD, categorized under frontotemporal lobar degeneration (FTLD) and classified by the presence of abnormal components of neuronal and glial inclusions. Some of the major subtypes include microtubuleassociated protein tau in FTLD-tau, TDP-43 in FTLD-TDP, and fused in sarcoma (FUS) protein in FTLD-FUS (14). FTLD-TDP is the most common pathological subtype, occurring in $\sim 50 \%$ of all cases. FTLD-tau accounts for $\sim 45 \%$ of all cases, followed by FTLD-FUS in $<5 \%$ of cases (13). The majority of individuals with FTLD-FUS are diagnosed with sporadic, early-onset bvFTD (6).

This report discusses the clinical presentation and genetic findings of an individual with early-onset FTD. Collectively, FTD is clinically heterogeneous, making it difficult to predict the underlying pathological or genetic processes. Thus, with these findings we aim to further understand the molecular pathology and systemic features of early-onset forms of FTD.

\section{CASE REPORT}

The patient was in good baseline health until age 27 years, when her family noticed an increase in aggression and childlike behaviors. Initially, a family member brought her to the emergency department for behaving erratically. She was later discharged and diagnosed with adjustment disorder with mixed emotional features.
Four months later, the patient was brought into the emergency department (ED) with suspicion of a psychiatric disorder. On exam, she was noticeably withdrawn and inattentive. Due to apparent psychosis, she was placed on hold and hospitalized. Following admission, she was given a provisional diagnosis of bipolar disorder type 1. She was placed on oral olanzapine and observed over three days with slight improvements in symptoms allowing for voluntary discharge.

The patient was brought to the ED again two months later after a physical altercation. In the ED, her psychiatric exam was notable for disorganized thoughts, mood lability, poor insight, and rapid, tangential speech; however, she was not agitated or aggressive. She was admitted on a 72-h hold for mania and psychosis. Her drug screen was negative and she was admitted to the psychiatry service. She did not respond to multiple antipsychotic medications or neuroleptic drugs. For workup of medically refractory psychosis, she completed a brain MRI which showed bilateral medial frontal, anterior temporal, and caudate head atrophy (Figure 1A). Clinicians in the neurology department then evaluated her and transferred her to their service for further testing.

She completed an EEG, MRI, and lumbar puncture. The EEG was characterized by diffuse theta/delta slowing consistent with non-specific encephalopathy of unknown etiology. Her second MRI, two months after initial MRI, showed similar changes to the prior MRI. The cerebrospinal fluid workup for infectious and autoimmune conditions was unrevealing. The cerebrospinal fluid autoantibody panel inclued NMDA-R, CASPR2-lgG, GAD65, ANNA-3, AGNA-1, PCA-Tr, Amphiphysin, VGKCComplex, LGI1-lgG, GABA-B-R, AMPA-R, ANNA-1, ANNA2, PCA-1, PCA-2, and CRMP-5-IgG. The blood autoantibody panel included NMDA-R, CASPR2-lgG, GAD65, GABA-BR, AMPA-R, ANNA-1, ANNA-2, ANNA-3, AGNA-1, PCATr, Amphiphysin, ACh Receptor (Muscle) Binding, AChR Ganglionic Neuronal, Neuronal (V-G) K+ Channel, LGI1-lgG, PCA-1, PCA-2, N-Type Calcium Channel, P/Q-Type Calcium Channel, and CRMP-5-IgG. After completing these exams, she was readmitted to the psychiatry department.

Three weeks later, a fluorodeoxyglucose (FDG)-positron emission tomography (PET) scan was performed, which showed hypometabolism that was severe in the orbitofrontal and caudate regions, moderate in the frontotemporal regions, and mild to moderate in the parietal and posterior cingulate regions (Figure 2). FDG-PET from skull to thigh revealed no evidence of malignancy. In the following months, her behavioral disinhibition and psychosis did not improve on multiple antipsychotic medications. She was administered six courses of electroconvulsive therapy over two weeks but showed no improvement.

Although a primary psychiatric illness was considered on initial presentation, her clinical features, lack of response to antipsychotics, and prominent atrophy seen via brain imaging indicated a neurodegenerative condition. The brain MRI and FDG-PET findings, along with her clinical features were consistent with behavioral variant FTD (bvFTD) (14). Her presentation was notable for lack of insight, diminished empathy, aberrant motor behaviors, verbal aggression, stereotyped phrases, 
A
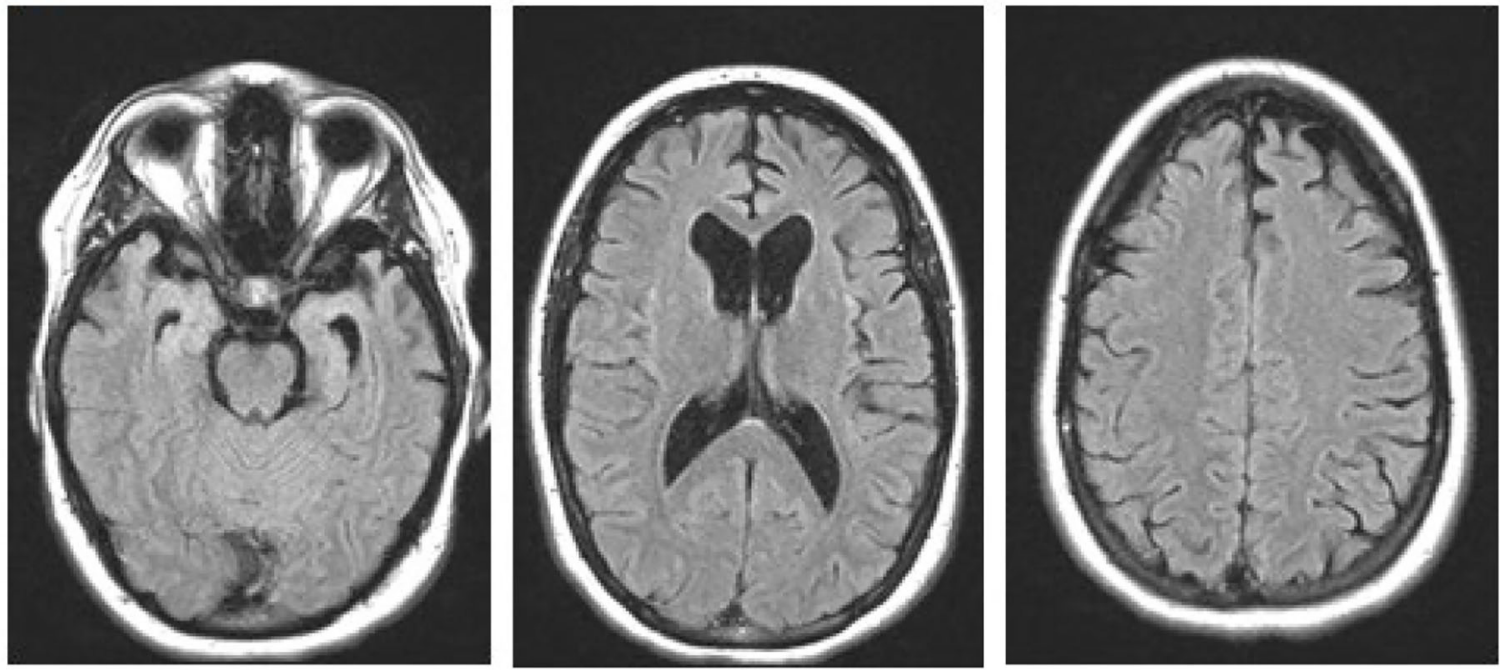

B
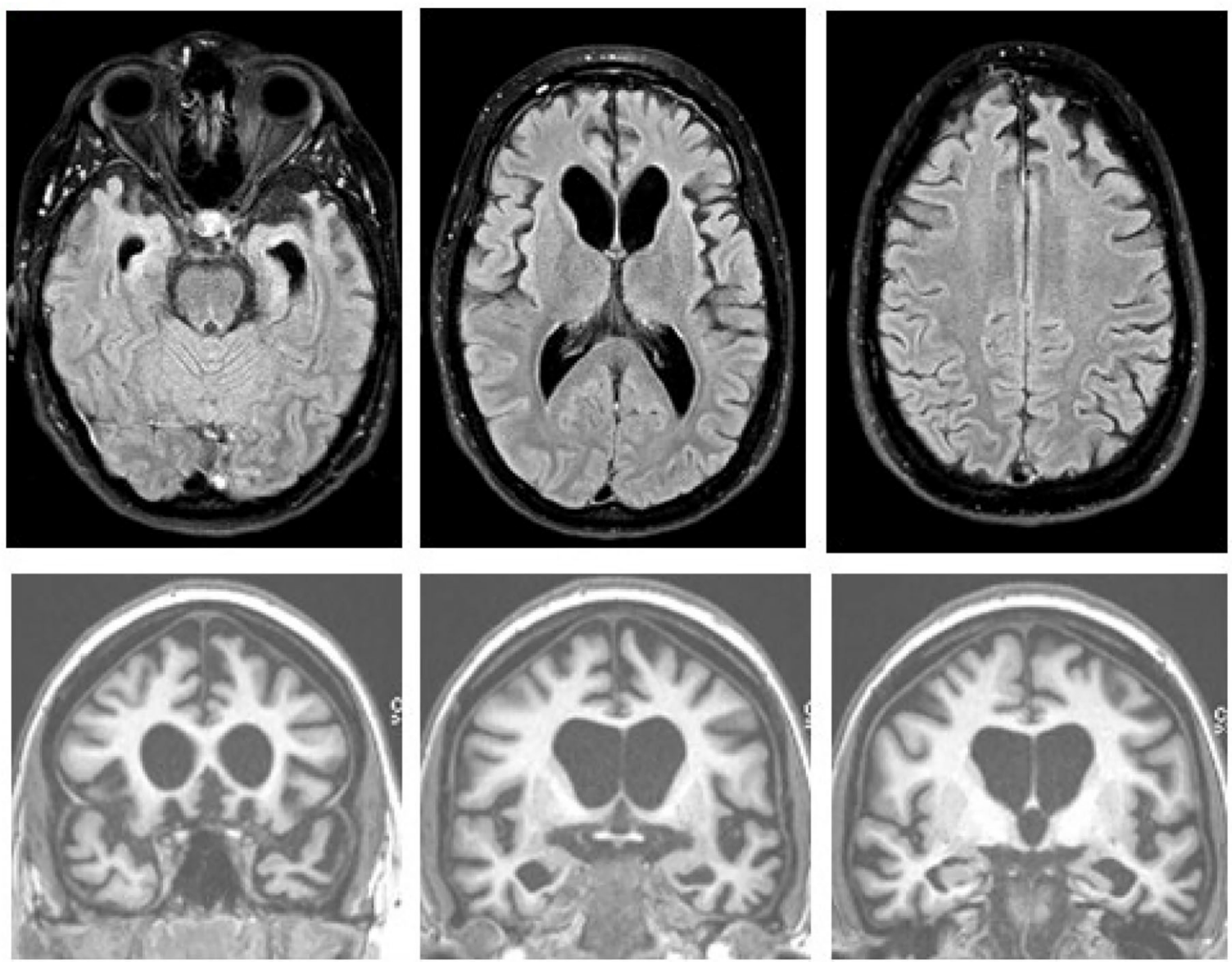

FIGURE 1 | Brain MR images at age 27 (A) and 19 months later (B), with axial fluid attenuation inversion recovery (FLAIR) images (top and middle rows) and coronal T1-weighted images (bottom row). Note progression of the bilateral medial frontal, anterior temporal, and caudate head atrophy with the associated ventricular enlargement on the axial FLAIR images. The orbitofrontal as well as dorsomedial, anterior temporal, and caudate head atrophy are obvious on the coronal images. Images are in standard radiology orientation. 


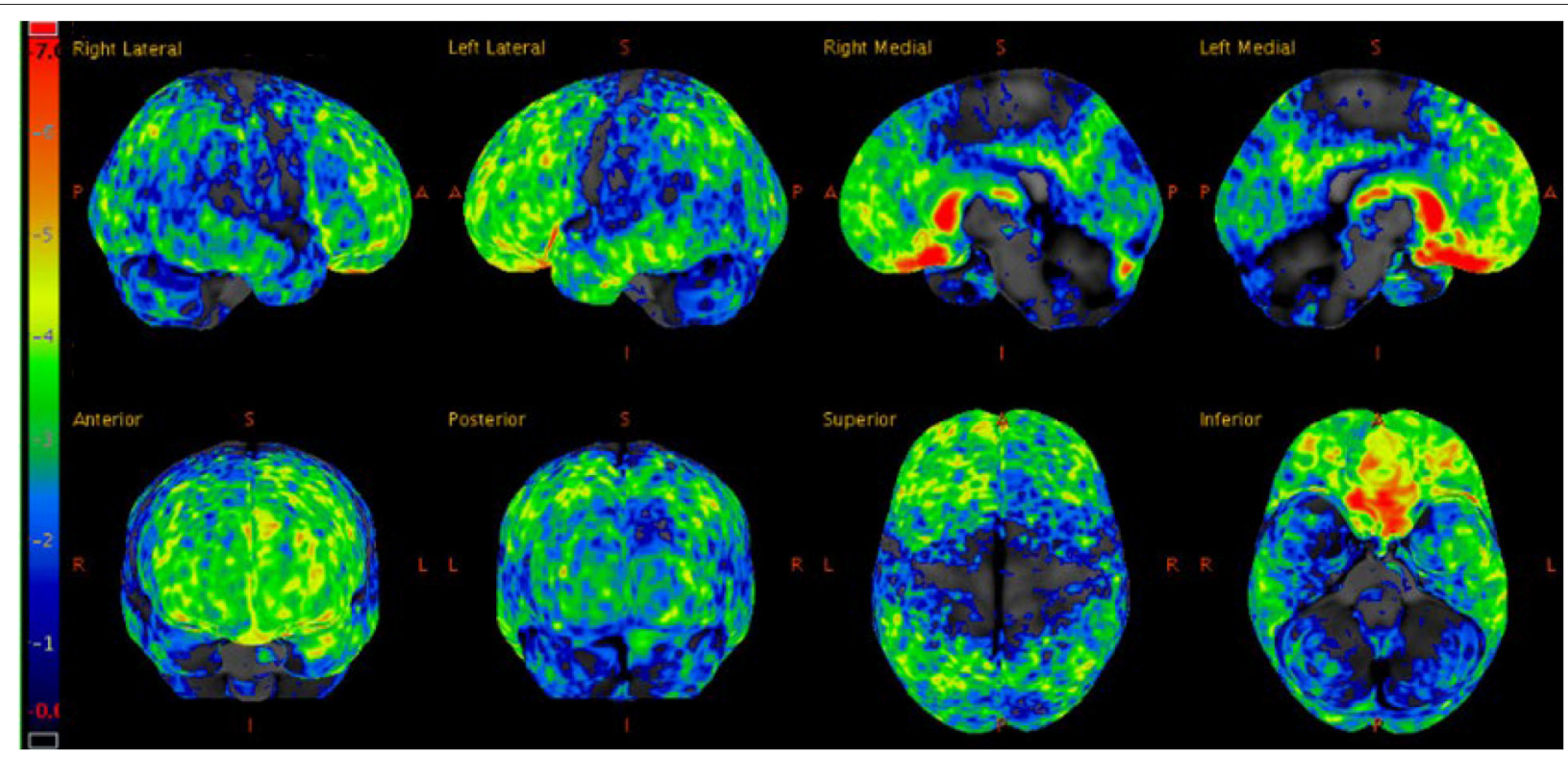

FIGURE 2 | Statistical z-score maps of brain FDG-PET at age 27, with the degree of hypometabolism reflected by the color bar on the left; dark blue is within normal limits and red is maximally abnormal. Note the degree of hypometabolism is severe in the orbitofrontal and caudate regions, moderate in the frontotemporal regions (left slightly more so than right), and mild to moderate in the parietal and posterior cingulate regions.

social withdrawal, disinhibition, and environmental dependence. There was no clinical evidence of motor or neuromuscular dysfunction. A five generation family history was reviewed and, with the exception of one second-degree relative with suspected mild cognitive impairment, two second-degree relatives with developmental delay, and one third-degree relative with arachnoid cyst, no other contributory or signficant family history was noted. Given the extremely young age and aggressive behavior with maintained verbal fluency, lack of family history of a neurodegenerative disease, and imaging findings particularly with prominent caudate atrophy/hypometabolsim, bvFTD with FUS proteinopathy was considered a strong possibility. MRI performed 19 months after the initial scan showed a progression of the medial frontal, anterior temporal, and caudate head atrophy with the associated ventricular enlargement (Figure 1B). No restricted diffusion was seen on this MRI or previous MRIs.

\section{GENETIC ANALYSIS}

Due to the unique presentation, we performed next generation sequencing (NGS) to analyze a panel of 28 genes associated with dementia and ALS. The panel included ALS2, APP, CHCHD10, CHMP2B, PCTN1, FUS, GRN, HNRNPA2B1, MAPT, MATR3, OPTN, PFN1, DRNP, PSEN1, PSEN2, PSG11, SETX, SIGMAR1, SNCA, SOD1, SQSTM1, TARDBP, TBK1, TFG, TREM2, UBQLN2, VAPB, and VCP. Genetic analysis initially indicated a duplication of exon 15 from the MATR3 gene in the patient. NGS of both parents' DNA revealed this same feature in one clinically unaffected parent. All other gene tests in this panel were negative. We also assessed for hexanucleotide repeat expansion in C9orf72 in the proband using a PCR based assay, and this test was normal $(<20)$. In addition, we did clinical whole exome sequencing on the patient and the parents to evaluate for any other possible causes of FTD, which was performed using the Agilent SureSelect NovaSeq instrument with average coverage across the entire capture of $180 \mathrm{x}$. The clinical whole exome sequence was negative for the patient and the parents.

We obtained blood samples from the patient and the parents to further characterize the MATR3 mutation that was identified through NGS. We performed whole genome sequencing (WGS) through the Genomics Center as follows. Three TruSeq unique dual-indexed DNA libraries were created. All libraries were pooled and sequenced on a NovaSeq 6000 system, with S2-type flow cell and run mode of $2 \times 150 \mathrm{bp}$. $\sim 300$ million pass-filter reads or greater were generated for each sample. Mean quality scores for all libraries were $\geq \mathrm{Q} 30$ indicating $<0.1 \%$ error rate in base calling. WGS indicated that variant 5 MATR3 cDNA (MATR3V5) had been inserted into chromosome 12 (CH12), flanked by 15 base-pair repeats, with an insertion site $20 \mathrm{kbp}$ upstream of the LIM Homeobox 5 (LHX5) gene. WGS results refuted the presence of an exon 15 duplication on chromosome 5 , which was not detected. Huntington's disease was ruled out by WGS indicating that there was no evidence for HTT CAG repeat expansion in any of the samples.

We verified the results of WGS using Phusion HighFidelity PCR (Thermo-Fisher) with primers crossing putative CH12/MATR3V5 junctions. Two independent assays were completed: assay 1 [forward primer (CH12F1), TCTCTGCTG GCTCTACCTAAA; reverse primer (MATR3e15-16R1), AGT TCCTCGATCTTGTCCACC], and assay 2 [forward primer (MATR3e16F1), TGAGAACGCTGATGATCCCAA; reverse 
primer (CH12R1), AAAAAGGTGTTTCCTGGGAGCG], which targeted the $5^{\prime}$ and $3^{\prime}$ ends of the insertion, respectively (Figure 3A). Presence of DNA was confirmed and band size was measured using UV/ethidium bromide visualization in $1 \%$ agarose gel. The mutation was confirmed in the patient and one healthy parent (Figure 3B). To determine whether this mutation could have arisen de novo in the unaffected parent, blood and serum samples were collected from both grandparents biologically related to the healthy parent carrier. Targeted PCR revealed that one healthy grandparent also carries the MATR3V5 insertion in CH12 (Figure 3B). These results indicate that, despite the MATR3V5 mutation being present in multiple generations, only the patient showed clinical symptoms.

To search for MATR3 transcripts in blood, total RNA was isolated using the PAXgene Blood RNA Kit for in vitro diagnostics (Qiagen, University of Minnesota Genomics Core). RNA integrity numbers were 7.4, 7.7, and 8.4 for the patient, parent, and grandparent, respectively, indicating minimally-degraded RNA. Using RT-PCR SuperScript III Reverse Transcriptase (Thermo-Fisher), a cDNA template was created for the proband, parents, and grandparents. First-strand synthesis was done using a primer near the $3^{\prime}$ end of MATR3V5 sequence (CCAAATGAAAGTCTGCAAGGCTCA). For PCR, primers were designed to detect MATR3 variants 1-5. However, no primer pairs produced detectable cDNA.

\section{DISCUSSION}

We present a case of an aggressive form of early-onset FTD. The patient exhibited many of the core features of bvFTD, including behavioral changes, verbal aggression, lack of empathy, stereotyped behaviors, and social withdrawal (15). Brain imaging showed significant atrophy in the frontal and anterior temporal lobes plus heads of the caudate, as well as hypometabolism maximal in bilateral frontal and temporal lobes and basal ganglia, consistent with bvFTD. This case presents a novel genetic mutation that may be linked with early-onset bvFTD. In the patient, one parent, and one grandparent, we discovered an insertion of MATR3V5 cDNA with flanking $15 \mathrm{bp}$ repeats. However, second- and third-degree relatives were asymptomatic. The clinical symptoms observed in this case do not align with existing mutations in MATR3 associated with early-onset bvFTD, most of which are point mutations (16). This case is unique because of the patient's atypical clinical presentation as early-onset bvFTD associated with a mechanism of mutation that has not been linked to bvFTD.

The insertion into chromosome 12 of the MATR3V5 transcript likely occurred through retrotransposition. MATR3V5 is a rare splice variant of the MATR3 gene native to chromosome 5 (17). Some point mutations in MATR3-S85C, F115C, and T622A-are associated with FTD-ALS spectrum pathology as well as distal myopathy and pharyngeal weakness $(17,18)$, but retrotransposition of MATR3V5 has, to the best of our knowledge, never been associated with bvFTD. Since MATR3 mutations have previously been linked to FTD-ALS spectrum, it is possible that the novel MATR3 retrotransposition found in this case is causally linked to the observed neurodegeneration.

Often, retrotransposition is benign and associated with evolutionary genetic expansion and gene regulation (19). However, disruptive retrotransposition has been linked to neurological diseases such as Aicardi-Goutières syndrome, multiple sclerosis, ALS, and Alzheimer's disease (20, 21). For example, increased levels of TDP-43, the most common pathological biomarker in FTD-ALS spectrum, leads to loss of post-transcriptional gene silencing responsible for retrotransposable element (RTE) repression $(21,22)$. Increased activity of RTEs in TDP-43 mediated diseases leads to disruptive retrotransposition, indicated by DNA damage/DNA nicking, and subsequently results in apoptosis (21). Although disruptive retrotransposition has been observed in FTD-ALS, the MATR3 retrotransposition in the proband's case is unlikely to be a consequence of neurodegeneration since it is present in healthy carriers.

In most cases of FTD-ALS, TDP-43 is the primary component of ubiquitinated-positive cytoplasmic inclusions where the C-terminal fragments of TDP-43 are ubiquitinated and phosphorylated $(5,21,22)$. In HEK293-FT cells, endogenous TDP-43 has been shown to co-immunoprecipitate with MATR3 protein (16). Further, the S85C mutant of MATR3 showed increased binding to TDP-43 compared to wild-type MATR3, but this effect was not seen in F115C and T622A mutants (16). Investigation of TDP-43's interaction with MATR3 may shed light on the mechanisms of FTD-ALS pathogenesis, though it remains unclear how retrotransposition of MATR3V5 into $\mathrm{CH} 12$, and potential aberrant expression of MATR3V5, impacts cellular function.

In a small number of FTD-ALS cases, FUS, another DNA/RNA binding protein, is the primary pathological protein involved (23). A missense mutation in the FUS gene has recently been identified as a cause of familial ALS. A mutation in FUS has also been linked to tau-negative and TDP-43-negative FTLD subtypes (13). In addition, mutations in FUS have been associated with MATR3 and TDP-43 cytoplasmic aggregation $(24,25)$. Complex interactions amongst proteins implicated in FTD-ALS indicate numerous pathways by which MATR3 dysfunction may cause cognitive deficits. Further study of MATR3's interactome is crucial to understanding its mechanism in FTD.

MATR3 modulation emulates aspects of FTD-ALS pathology in vitro. Elevated levels of wild-type MATR3 and MATR3 mutants (S85C, F115C, P154S, and T622A) elicit a dosedependent toxicity in primary rat mixed cortical cultures. Similarly, siRNA knockdown of endogenous MATR3 also results in greater cell death compared to non-targeting siRNA. These results illustrate that both increased and decreased neuronal MATR3 leads to increased cell death, exemplifying that the mechanism of MATR3 in FTD-ALS pathology may be a gain or loss of function, or both (11).

It is unclear whether the MATR3V5 retrotransposition on CH12 encodes an mRNA or produces a protein. MATR3 expression in the blood is low, and attempts to isolate mRNA or protein from blood and serum samples have been largely unsuccessful. Transcription of MATR $3 V 5$ from 
A

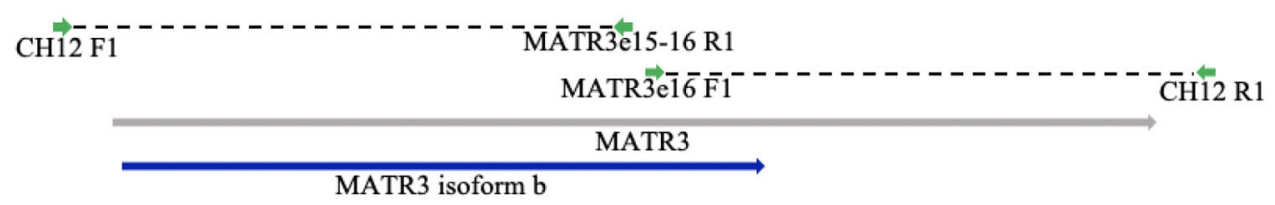

MATR3 isoform b

CH12 insertion site of MATR3 pseudogene

5 ' end of MATR 3 retrotransposition

$15 \mathrm{bp}$ direct repeat flanking pseudogene

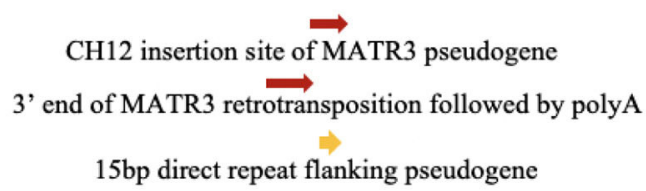

$15 \mathrm{bp}$ direct repeat flanking pseudogene

B Assay 1 Assay 2

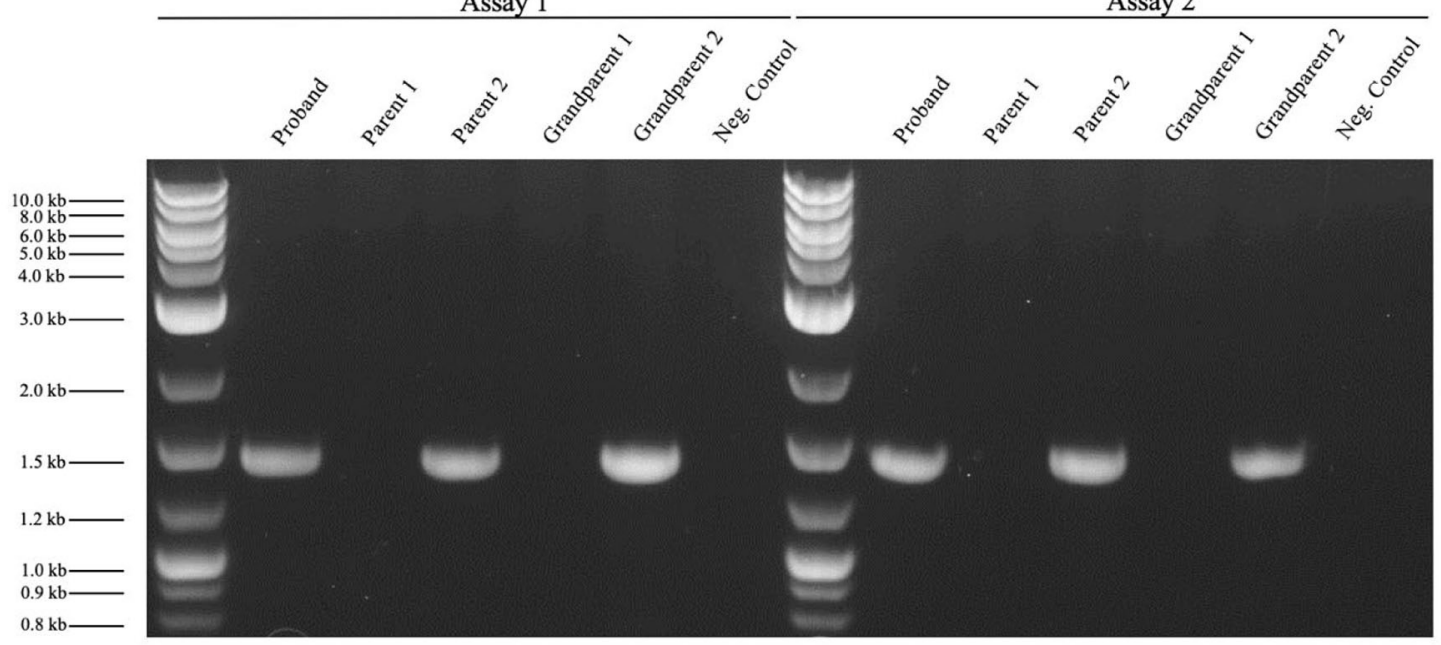

FIGURE 3 | Targeted PCR confirmed the MATR3V5 insertion in chromosome 12. (A) Diagram of chromosome 12 insertion site of MATR3 variant 5 and primers used for PCR. MATR3 gene sequence that codes for the matrin-3 protein isoform b is indicated in blue. (B) Patient (proband), parental, and grandparental genomic DNA were amplified by targeted PCR. Genomic DNA from the patient, parent 2, and grandparent 2 carried the mutation found in chromosome 12. A 1 kbp ladder was used in lanes 1 and 8 . The expected product sizes were 1,457 bp for Assay 1 and 1,435 bp for Assay 2. Parent 1's genomic DNA was used for the negative control which lacked DNA polymerase.

CH12 could conceivably increase or decrease MATR3 protein levels by subsequent translation or RNA-silencing, respectively. MATR3V5 mRNA from CH5 codes for the MATR3 isoform b protein. Isoform a, translated from variants $1-4$, is 847 amino acids in length while, isoform $b$ translated from variant 5, is 559 amino acids and shorter on the $\mathrm{N}$ terminus. Both isoforms are DNA/RNA binding proteins and carry the same main domains $(11,26)$. Determination of the functional outcome of CH12-MATR3V5 transcription requires proteinlevel analysis.

In future studies, it would be informative to culture forebrain neurons with this mutation using patient derived induced pluripotent stem cells (iPSCs), as this provides a more direct means to study differential localization, altered transcription and translation levels, and mechanisms of MATR3. Notably, MATR3V5 is located $20 \mathrm{kbp}$ downstream of the LHX5 gene on CH12. LHX5 encodes a protein family that function as transcriptional regulators of neuronal differentiation, migration, and development of the forebrain. Because of its chromosomal proximity to a gene active in the cortex and hippocampus, it will be worth studying how the MATR3V5 insertion may affect-or be impacted by-the LHX5 locus. In addition, MATR3 cellular localization can be studied using iPSCs. MATR3 localization has been shown to play a role in neurotoxicity. Cytoplasmic MATR3 redistribution from the nucleus extends survival in neurons that overexpress MATR3, suggesting that nuclear MATR3 mediates neurotoxicity (11). Epigenetic differences between the patient and the unaffected carriers could also result in differences in expression of the MATR3 retrotransposed gene. Collectively, these analyses will allow a thorough investigation of the MATR3 protein and transcript and its role in the pathology of earlyonset FTD.

This case study highlights a novel gene retrotransposition of MATR3V5 associated with a rare case of early-onset bvFTD. The results of this case study illustrate that point mutations may not be the only genetic mechanism by which MATR3 contributes to FTD. These findings also add to 
our knowledge of potentially active pseudogenes contributing to dementia pathologies. Future studies will investigate the significance of this gene retrotransposition, potential role of MATR3V5 in disease, and its association with the earlyonset bvFTD pathology presented in this case. Moreover, a thorough understanding of this novel MATR3 mutation may help elucidate mechanisms of matrin-3 dysregulation in other neurodegenerative contexts.

\section{DATA AVAILABILITY STATEMENT}

The raw data supporting the conclusions of this article will be made available by the authors, without undue reservation.

\section{ETHICS STATEMENT}

This study was approved by the University of Minnesota's institutional review board. All procedures performed during this study involving human participants were in accordance with the ethical standards of the institutional review board at the University of Minnesota and with the 1964 Helsinki Declaration and its later amendments or comparable ethical standards. Surrogate consent was received for the participant. Informed consent was obtained from all other individuals able to independently consent. Written informed consent was

\section{REFERENCES}

1. Merrilees J, Klapper J, Murphy J, Lomen-Hoerth C, Miller BL. Cognitive and behavioral challenges in caring for patients with frontotemporal dementia and amyotrophic lateral sclerosis. Amyotroph Lateral Scler. (2010) 11:298302. doi: $10.3109 / 17482961003605788$

2. Onyike CU, Diehl-Schmid J. The epidemiology of frontotemporal dementia. Int Rev Psychiatry. (2013) 25:130-7. doi: 10.3109/09540261.2013.776523

3. Lanata SC, Miller BL. The behavioural variant frontotemporal dementia (bvFTD) syndrome in psychiatry. J Neurol Neurosurg Psychiatry. (2016) 87:501-11. doi: 10.1136/jnnp-2015-310697

4. Goldman JS. Genetic testing and counseling in the diagnosis and management of young-onset dementias. Psychiatr Clin North Am. (2015) 38:295308. doi: 10.1016/j.psc.2015.01.008

5. Neumann M, Tolnay M, Mackenzie IR. The molecular basis of frontotemporal dementia. Expert Rev Mol Med. (2009) 11:e23. doi: 10.1017/S1462399409001136

6. Liu MN, Lau CI, Lin CP. Precision medicine for frontotemporal dementia. Front Psychiatry. (2019) 10:75. doi: 10.3389/fpsyt.2019.00075

7. Goldman JS, Farmer JM, Van Deerlin VM, Wilhelmsen KC, Miller BL, Grossman M. Frontotemporal dementia: genetics and genetic counseling dilemmas. Neurologist. (2004) 10:22734. doi: 10.1097/01.nrl.0000138735.48533.26

8. Goldman JS, Van Deerlin VM. Alzheimer's disease and frontotemporal dementia: the current state of genetics and genetic testing since the advent of next-generation sequencing. Mol Diagn Ther. (2018) 22:50513. doi: 10.1007/s40291-018-0347-7

9. Seelaar H, Rohrer JD, Pijnenburg YA, Fox NC, van Swieten JC. Clinical, genetic and pathological heterogeneity of frontotemporal dementia: a review. J Neurol Neurosurg Psychiatry. (2011) 82:476-86. doi: 10.1136/jnnp.2010.212225

10. Ramos EM, Dokuru DR, Van Berlo V, Wojta K, Wang Q, Huang AY, et al. Genetic screening of a large series of North American sporadic and familial frontotemporal dementia cases. Alzheimers Dement. (2020) 16:11830. doi: 10.1002/alz.12011 obtained from the participant's authorized representative for the publication of any potentially identifiable images or data included in this article.

\section{AUTHOR CONTRIBUTIONS}

MC and KV obtained IRB approval. MC, NV, and KV drafted the manuscript. KV and BB examined the patient. DD, MB, and $\mathrm{MK}$ completed the genetic analysis. NV completed the PCR assays. SP provided guidance and technical support for PCR assays. All authors reviewed and revised the final manuscript.

\section{FUNDING}

This study was supported by funds from the N. Bud Grossman Center for Memory Research and Care and the Institute for Translational Neuroscience at the University of Minnesota.

\section{ACKNOWLEDGMENTS}

We express our sincere thanks to the patient and her family members. We thank the University of Minnesota Genomics Center for whole genome sequencing. We thank Kasey Ah Pook for his contribution in obtaining IRB approval and editing the manuscript.

11. Malik AM, Miguez RA, Li X, Ho YS, Feldman EL, Barmada SJ. Matrin 3-dependent neurotoxicity is modified by nucleic acid binding and nucleocytoplasmic localization. Elife. (2018) 7:e35977. doi: 10.7554/eLife.35977

12. Boehringer A, Garcia-Mansfield K, Singh G, Bakkar N, Pirrotte P, Bowser R. ALS associated mutations in matrin 3 alter proteinprotein interactions and impede mrna nuclear export. Sci Rep. (2017) 7:14529. doi: 10.1038/s41598-017-14924-6

13. Mackenzie IR, Rademakers R, Neumann M. TDP-43 and FUS in amyotrophic lateral sclerosis and frontotemporal dementia. Lancet. Neurol. (2010) 9:9951007. doi: 10.1016/S1474-4422(10)70195-2

14. Bahia VS, Takada LT, Deramecourt V. Neuropathology of frontotemporal lobar degeneration: a review. Dement Neuropsychol. (2013) 7:19-26. doi: 10.1590/S1980-57642013DN70100004

15. Rascovsky K, Hodges JR, Knopman D, Mendez MF, Kramer JH, Neuhaus J, et al. Sensitivity of revised diagnostic criteria for the behavioural variant of frontotemporal dementia. Brain. (2011) 134:2456-77. doi: 10.1093/brain/awr179

16. Johnson JO, Pioro EP, Boehringer A, Chia R, Feit H, Renton AE, et al. Mutations in the matrin 3 gene cause familial amyotrophic lateral sclerosis. Nat Neurosci. (2014) 17:664-6. doi: 10.1038/nn.3688

17. Senderek J, Garvey SM, Krieger M, Guergueltcheva V, Urtizberea A, Roos A, et al. Autosomal-dominant distal myopathy associated with a recurrent missense mutation in the gene encoding the nuclear matrix protein, matrin 3. Am J Hum Genet. (2009) 84:511-8. doi: 10.1016/j.ajhg.2009.03.006

18. Gallego-Iradi MC, Clare AM, Brown HH, Janus C, Lewis J, Borchelt DR. Subcellular localization of matrin 3 containing mutations associated with als and distal myopathy. PLoS ONE. (2015) 10:e0142144. doi: 10.1371/journal.pone.0142144

19. Janousek V, Laukaitis CM, Yanchukov A, Karn RC. The role of retrotransposons in gene family expansions in the human and mouse genomes. Genome Biol Evol. (2016) 8:2632-50. doi: 10.1093/gbe/evw192

20. Tam OH, Ostrow LW, Gale Hammell M. Diseases of the nERVous system: retrotransposon activity in neurodegenerative disease. Mob DNA. (2019) 10:32. doi: 10.1186/s13100-019-0176-1 
21. Krug L, Chatterjee N, Borges-Monroy R, Hearn S, Liao WW, Morrill K, et al. Retrotransposon activation contributes to neurodegeneration in a drosophila TDP-43 model of ALS. PLoS Genet. (2017) 13:e1006635. doi: 10.1371/journal.pgen.100 6635

22. Majumder V, Gregory JM, Barria MA, Green A, Pal S. TDP-43 as a potential biomarker for amyotrophic lateral sclerosis: a systematic review and metaanalysis. BMC Neurol. (2018) 18:90. doi: 10.1186/s12883-018-1091-7

23. Ling SC, Polymenidou M, Cleveland DW. Converging mechanisms in ALS and FTD: disrupted RNA and protein homeostasis. Neuron. (2013) 79:41638. doi: 10.1016/j.neuron.2013.07.033

24. Yamaguchi A, Takanashi K. FUS interacts with nuclear matrix-associated protein SAFB1 as well as Matrin3 to regulate splicing and ligandmediated transcription. Sci Rep. (2016) 6:35195. doi: 10.1038/srep3 5195

25. Kamelgarn M, Chen J, Kuang L, Arenas A, Zhai J, Zhu H, et al. Proteomic analysis of FUS interacting proteins provides insights into FUS function and its role in ALS. Biochim Biophys Acta. (2016) 1862:200414. doi: 10.1016/j.bbadis.2016.07.015

26. Belgrader P, Dey R, Berezney R. Molecular cloning of matrin 3. A 125 kilodalton protein of the nuclear matrix contains an extensive acidic domain. J Biol Chem. (1991) 266:9893-9.

Conflict of Interest: The authors declare that the research was conducted in the absence of any commercial or financial relationships that could be construed as a potential conflict of interest.

Copyright (C) 2020 Castro, Venkateswaran, Peters, Deyle, Bower, Koob, Boeve and Vossel. This is an open-access article distributed under the terms of the Creative Commons Attribution License (CC BY). The use, distribution or reproduction in other forums is permitted, provided the original author(s) and the copyright owner(s) are credited and that the original publication in this journal is cited, in accordance with accepted academic practice. No use, distribution or reproduction is permitted which does not comply with these terms. 